\title{
A NOTE ON WIENER-HOPF DETERMINANTS AND THE BORODIN-OKOUNKOV IDENTITY
}

\author{
Estelle L. Basor' and Yang Chen
}

\begin{abstract}
The continuous analogue of a Toeplitz determinant identity for Wiener-Hopf operators is proved. An example which arises from random matrix theory is studied and an error term for the asymptotics of the determinant is computed.
\end{abstract}

\section{Wiener-Hopf Determinants}

Recently, a beautiful identity due to Borodin and Okounkov was proved for Toeplitz determinants which shows how one can write a Toeplitz determinant as a Fredholm determinant. In this note we generalize this to the Wiener-Hopf case. The proof in the Wiener-Hopf case follows identically with the second one given in [1]. We include it here for completeness sake and because the nature of the identity is slightly different in the continuous verses discrete convolution setting.

In the Wiener-Hopf case we begin with a Fredholm determinant on a finite interval and then show how this can be written as a Fredholm determinant of an operator defined on $L^{2}$ of a half-line. The point is the second operator has a very "small" kernel and thus higher order approximations (as a function of the length of the finite interval) can be found.

We now state the analogue of the identity and then apply it to a particular case to show how error estimates can be computed. In the future we hope to refine the estimates given here, apply this identity to other important examples, and also extend it to other operators.

We consider the Fredholm determinant of the finite Wiener-Hopf operator

$$
\operatorname{det}\left(I-\mathcal{K}_{[0, \alpha]}\right)
$$

\footnotetext{
${ }^{1}$ Supported by National Science Foundation grant DMS-9970879 and EPSRC Grant GR/N35281.
} 
where the $\mathcal{K}$ acts on $L^{2}(0, \alpha)$ and has kernel $\mathcal{K}(x-y)$ with $\mathcal{K}$ given by the Fourier transform of a function $F$, i.e.

$$
F(\xi)=\int_{-\infty}^{\infty} \mathcal{K}(x) e^{i \xi x} d x .
$$

The continuous analogue of the Borodin-Okounkov identity is given, under appropriate conditions by the formula

$$
\operatorname{det}\left(I-\mathcal{K}_{[0, \alpha]}\right)=Z e^{c \alpha} \operatorname{det}\left(I-\mathcal{L}_{[\alpha, \infty)}\right),
$$

where

$$
c:=\int_{-\infty}^{\infty} \ln (1-F(\xi)) \frac{d \xi}{2 \pi},
$$

$\mathcal{L}$ is an operator acting on $L^{2}[\alpha, \infty)$ with kernel,

$$
\mathcal{L}(x, y)=\int_{0}^{\infty}\left(\frac{\phi_{-}}{\phi_{+}}-1\right)_{x+z}\left(\frac{\phi_{+}}{\phi_{-}}-1\right)_{-z-y} d z,
$$

and $Z$ is a certain constant whose value will be defined shortly. Here $\phi_{x}$ is inverse Fourier transform of $\phi(\xi)$, and $\phi_{ \pm}(\xi)$ are the Wiener-Hopf factors satisfying;

$$
1-F(\xi)=\phi_{+}(\xi) \phi_{-}(\xi), \quad \xi \in \mathbf{R},
$$

and also satisfying the condition that the functions $\phi_{ \pm}$when extended away from $\mathbf{R}$ are analytic in the upper and lower half-plane respectively. So that this makes sense and our proof is valid we require that $F$ is bounded and in $L^{1}(\mathbf{R})$ and that $\mathcal{K}$ is also in $L^{1}(\mathbf{R})$ and satisfies $\int_{-\infty}^{\infty}|x||\mathcal{K}|(x) d x<\infty$. To achieve an unique factorization, $1-F$ must have index zero, be bounded away from zero and we assume that $\phi_{ \pm}$are one at $\pm \infty$. These conditions guarantee that the operator $A-I$ in the proof below is trace class and also that all the integrals defined are finite. For details see 四.

In this section we include the proof of the identity as promised. In the next section we apply it to a particular example that arises in random matrix theory [8]. In this example

$$
\mathcal{K}(x-y):=\frac{g \sin \pi(x-y)}{\pi \sinh g(x-y)}, \quad x, y \in \mathbf{R}, \quad g>0 .
$$

We show that as $\alpha \rightarrow \infty$,

$$
\operatorname{det}\left(I-\mathcal{L}_{[\alpha, \infty)}\right) \sim 1-C(g) \mathrm{e}^{-2 g \alpha(1-\theta / \pi)},
$$

where $C(g)$ is a completely determined constant and $\cos \theta:=e^{-\pi^{2} / g}, \quad 0<\theta<\pi / 2$. This is a refinement of the classical Szegö-Kac-Widom Theorem. Previous attempts at the refinement computed the exponential term but not the constant. In principal, as the reader will see, higher order terms can also be found using the method outlined in section two. 
Here is a proof of the above identity in the Wiener-Hopf case. As already stated this proof follows from [1]. The interested reader should also note another slightly different and very

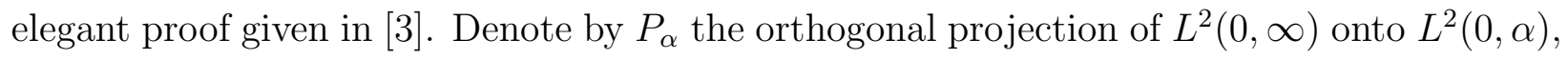
$Q_{\alpha}=I-P_{\alpha}$, and $P$ the orthogonal projection of $L^{2}(-\infty, \infty)$ onto $L^{2}(0, \infty)$. Also define $W(\phi)$ to be $P M_{\phi} P$ and $W_{\alpha}(\phi)$ to be $P_{\alpha} M_{\phi} P_{\alpha}$ where $M_{\phi}$ is multiplication by $\phi$. Note that by using Fourier transforms it can be proved that $W_{\alpha}(\phi)$ is unitarily equivalent to $I-\mathcal{K}_{[0, \alpha]}$ with $\phi=1-F$. It is straight-forward to check that

$$
P_{\alpha} W\left(\phi_{+}\right)=P_{\alpha} W\left(\phi_{+}\right) P_{\alpha}, \quad W\left(\phi_{-}\right) P_{\alpha}=P_{\alpha} W\left(\phi_{-}\right) P_{\alpha},
$$

and

$$
W\left(\phi_{+}\right) W\left(\phi_{+}^{-1}\right)=I, \quad W\left(\phi_{-}\right) W\left(\phi_{-}^{-1}\right)=I .
$$

Using the above, we can write ${ }^{2}$

$$
\begin{gathered}
P_{\alpha} W(\phi) P_{\alpha}=P_{\alpha} W\left(\phi_{+}\right) W\left(\phi_{+}^{-1}\right) W(\phi) W\left(\phi_{-}^{-1}\right) W\left(\phi_{-}\right) P_{\alpha} \\
=P_{\alpha} W\left(\phi_{+}\right) P_{\alpha} W\left(\phi_{+}^{-1}\right) W(\phi) W\left(\phi_{-}^{-1}\right) P_{\alpha} W\left(\phi_{-}\right) P_{\alpha} .
\end{gathered}
$$

Now it can be shown that the product of the determinants of $P_{\alpha} W\left(\phi_{ \pm}\right) P_{\alpha}$ are equal to $e^{c \alpha}$, (see [4], section 10.79). Thus to compute $\operatorname{det}\left(I-\mathcal{K}_{[0, \alpha]}\right)$ we need to consider $P_{\alpha} W\left(\phi_{+}^{-1}\right) W(\phi) W\left(\phi_{-}^{-1}\right) P_{\alpha}$.

Set

$$
W\left(\phi_{+}^{-1}\right) W(\phi) W\left(\phi_{-}^{-1}\right)=A .
$$

Notice that the determinant of $P_{\alpha} A P_{\alpha}$ equals $\operatorname{det}\left(P_{\alpha} A P_{\alpha}+Q_{\alpha}\right)$. Now $A$ is invertible and differs from $I$ by a trace class operator [四]. Therefore

$$
\begin{gathered}
\operatorname{det}\left(P_{\alpha} A P_{\alpha}+Q_{\alpha}\right)=\operatorname{det} A \operatorname{det}\left(A^{-1} P_{\alpha} A P_{\alpha}+A^{-1} Q_{\alpha}\right) \\
=\operatorname{det} A \operatorname{det}\left(A^{-1}\left(I-Q_{\alpha}\right) A P_{\alpha}+A^{-1} Q_{\alpha}\right)=\operatorname{det} A \operatorname{det}\left(P_{\alpha}-A^{-1} Q_{\alpha} A P_{\alpha}+A^{-1} Q_{\alpha}\right) \\
=\operatorname{det} A \operatorname{det}\left(P_{\alpha}+A^{-1} Q_{\alpha}\right) \operatorname{det}\left(I-Q_{\alpha} A P_{\alpha}\right),
\end{gathered}
$$

since $P_{\alpha} Q_{\alpha}=0$. The determinant of the operator on the right equals one, again since $P_{\alpha} Q_{\alpha}=0$. Moreover

$$
\operatorname{det}\left(P_{\alpha}+A^{-1} Q_{\alpha} Q_{\alpha}\right)=\operatorname{det}\left(I-\left(I-A^{-1}\right) Q_{\alpha}\right)=\operatorname{det}\left(I-Q_{\alpha}\left(I-A^{-1}\right) Q_{\alpha}\right) .
$$

We have shown

$$
\operatorname{det}\left(I-\mathcal{K}_{[0, \alpha]}\right)=\operatorname{det} A \operatorname{det}\left(I-Q_{\alpha}\left(I-A^{-1}\right) Q_{\alpha}\right) .
$$

\footnotetext{
${ }^{2}$ It is an easy general fact that if $\psi_{1} \in \overline{H^{\infty}}$ or $\psi_{2} \in H^{\infty}$ then $W\left(\psi_{1} \psi_{2}\right)=W\left(\psi_{1}\right) W\left(\psi_{2}\right)$. In particular $W\left(\phi_{ \pm}\right)$are invertible with inverses $W\left(\phi_{ \pm}^{-1}\right)$. Recall that $H^{\infty}$ consists of all $\psi \in L^{\infty}$ such that the Fourier transform of $\psi$ vanishes on the negative real axis.
} 
It remains to show that this is the same as (1.3). First, $A$ is similar via the invertible operator $W\left(\phi_{+}\right)$to $W(\phi) W\left(\phi_{-}^{-1}\right) W\left(\phi_{+}^{-1}\right)$. Therefore

$$
\operatorname{det} A=\operatorname{det} W(\phi) W\left(\phi_{-}^{-1}\right) W\left(\phi_{+}^{-1}\right)=\operatorname{det} W(\phi) W\left(\phi^{-1}\right) .
$$

This is a representation of the constant $Z Z^{\mathrm{s}}$ in (1.3). Next

$$
\begin{gathered}
A^{-1}=W\left(\phi_{-}\right) W(\phi)^{-1} W\left(\phi_{+}\right)=W\left(\phi_{-}\right) W\left(\phi_{+}^{-1}\right) W\left(\phi_{-}^{-1}\right) W\left(\phi_{+}\right) \\
=W\left(\phi_{-} / \phi_{+}\right) W\left(\phi_{+} / \phi_{-}\right) .
\end{gathered}
$$

Because $\phi_{-} / \phi_{+}$and $\phi_{+} / \phi_{-}$are reciprocals it follows easily from the algebra properties of our operators that the determinant of

$$
\left(I-Q_{\alpha}\left(I-A^{-1}\right) Q_{\alpha}\right)
$$

is the same as the determinant given in the right-hand side of (3) and this completes the proof.

\section{An Example}

Next we turn to the example cited in the first section. Consider (1.1) with

$$
\mathcal{K}(x-y):=\frac{g \sin \pi(x-y)}{\pi \sinh g(x-y)}, \quad x, y \in \mathbf{R}, \quad g>0 .
$$

The Fourier transform is given by,

$$
F(\xi)=\frac{\sinh \left(\pi^{2} / g\right)}{\cosh \left(\pi^{2} / g\right)+\cosh (\pi \xi / g)} .
$$

It is clear that $F$ satisfies our hypothesis. Writing, $1-F(\xi)=\exp (\psi(\xi))$, and

$$
\psi(\xi)=\int_{0}^{\infty} \psi_{t} e^{i \xi t} d t+\int_{-\infty}^{0} \psi_{t} e^{i \xi t} d t
$$

where

$$
\psi_{t}=\int_{-\infty}^{\infty} \ln (1-F(\xi)) e^{-i \xi t} \frac{d \xi}{2 \pi}
$$

yields

$$
\frac{\phi_{ \pm}(\xi)}{\phi_{\mp}(\xi)}=\exp ( \pm i \Phi(\xi)),
$$

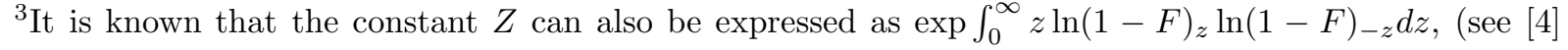
section 10.79).
} 
with

$$
\Phi(\xi)=2 i \int_{0}^{\infty} \psi_{t} \sin (\xi t) d t
$$

A simple calculation shows that

$$
\mathcal{L}(x, y)=\int_{0}^{\infty} f(x+z) f(z+y) d z
$$

where

$$
f(x):=\left(\frac{\phi_{+}}{\phi_{-}}-1\right)_{-x}=\int_{-\infty}^{\infty}\left(e^{i \Phi(\xi)}-1\right) e^{i \xi x} \frac{d \xi}{2 \pi}=\left(\frac{\phi_{-}}{\phi_{+}}-1\right)_{x} .
$$

To find $f(t)$ we need to compute $\psi_{t}$ and $\Phi(\xi)$. With $a:=e^{-\pi^{2} / g}$, a calculation gives,

$$
\psi_{t}=\frac{\cos (\pi t)-\cosh (g \theta t / \pi)}{t \sinh (g t)}, \quad a=: \cos \theta, \quad 0<\theta<\pi / 2 .
$$

It is a bit difficult to compute $\Phi(\xi)$ directly, so instead of finding $\Phi(\xi)$ we attempt to find its derivative. Using (2.18) and (2.21) and we find, that

$$
\Phi(\xi)=2 i \int_{0}^{\infty}\left(\frac{\cos (\pi t)-\cosh (g \theta t / \pi)}{t \sinh (g t)}\right) \sin (\xi t) d t .
$$

Next using the above and formula 3.524.5 of [2], gives the integral as a limit as $\mu \rightarrow 1$ of the sum of $\zeta(\mu,(1-\beta / \gamma) / 2)$ and $\zeta(\mu,(1+\beta / \gamma) / 2)$. Then using the definition of the $\zeta$ function, rearranging the sums, and finally letting $\mu \rightarrow 1$ we obtain

$$
\begin{aligned}
2 g \Phi^{\prime}(\xi) & =\Psi\left(\frac{1}{2}\left(1+\frac{\theta}{\pi}\right)-\frac{i \xi}{2 g}\right)-\Psi\left(\frac{1}{2}+\frac{i(\pi+\xi)}{2 g}\right) \\
& +\Psi\left(\frac{1}{2}\left(1+\frac{\theta}{\pi}\right)+\frac{i \xi}{2 g}\right)-\Psi\left(\frac{1}{2}+\frac{i(\pi-\xi)}{2 g}\right) \\
& +\Psi\left(\frac{1}{2}\left(1-\frac{\theta}{\pi}\right)+\frac{i \xi}{2 g}\right)-\Psi\left(\frac{1}{2}-\frac{i(\pi+\xi)}{2 g}\right) \\
& +\Psi\left(\frac{1}{2}\left(1-\frac{\theta}{\pi}\right)-\frac{i \xi}{2 g}\right)-\Psi\left(\frac{1}{2}-\frac{i(\pi-\xi)}{2 g}\right)
\end{aligned}
$$

where $\Psi$ is the di-gamma function. Integrating (2.23) with respect to $\xi$ with the initial condition $\Phi(0)=0$, gives,

$$
\begin{aligned}
\exp (i \Phi(\xi))= & \frac{\Gamma\left(\frac{1}{2}\left(1+\frac{\theta}{\pi}\right)+\frac{i \xi}{2 g}\right)}{\Gamma\left(\frac{1}{2}\left(1+\frac{\theta}{\pi}\right)-\frac{i \xi}{2 g}\right)} \frac{\Gamma\left(\frac{1}{2}\left(1-\frac{\theta}{\pi}\right)+\frac{i \xi}{2 g}\right)}{\Gamma\left(\frac{1}{2}\left(1-\frac{\theta}{\pi}\right)-\frac{i \xi}{2 g}\right)} \\
& \times \frac{\Gamma\left(\frac{1}{2}\left(1+\frac{i \pi}{g}\right)-\frac{i \xi}{2 g}\right)}{\Gamma\left(\frac{1}{2}\left(1-\frac{i \pi}{g}\right)+\frac{i \xi}{2 g}\right)} \frac{\left.\Gamma\left(1-\frac{i \pi}{g}\right)-\frac{i \xi}{2 g}\right)}{\Gamma\left(\frac{1}{2}\left(1+\frac{i \pi}{g}\right)+\frac{i \xi}{2 g}\right)} .
\end{aligned}
$$


We need now to compute (2.20). Note that the integrand vanishes when $\xi$ is zero. Furthermore, by using the asymptotics of the Gamma functions, it is easily seen that the integral converges conditionally. Putting $s=\frac{i \xi}{2 g}$ and $z=\exp (-2 g x)$, in (2.20), we find,

$$
\frac{f(x)}{2 g}=\int_{-i \infty}^{i \infty}\left(\frac{\Gamma(a+s)}{\Gamma(a-s)} \frac{\Gamma(1-a+s)}{\Gamma(1-a-s)} \frac{\Gamma(b-s)}{\Gamma(1-b+s)} \frac{\Gamma(1-b-s)}{\Gamma(b+s)}-1\right) z^{s} \frac{d s}{2 \pi i},
$$

where

$$
\begin{aligned}
a & =\frac{1}{2}\left(1+\frac{\theta}{\pi}\right), \\
b & =\frac{1}{2}\left(1+\frac{i \pi}{g}\right) .
\end{aligned}
$$

To compute the integral (2.25) we take a sequence of contours consisting of the line segment from $-i R_{n}$ to $i R_{n}$ and the semi-circle of radius $R_{n}$ in the left-half plane centered at the origin. Here $R_{n}=-n+\delta$, where $\delta$ is any fixed constant satisfying $0<\delta<1 / 4$. Now computing the residues of the Gamma functions in the left-half plane we obtain after taking the limit $n \rightarrow \infty$ the sum of two hypergeometric functions ${ }_{4} F_{3}$;

$$
\begin{aligned}
\frac{f(x)}{2 g}= & \left(\frac{\Gamma\left(\frac{\theta}{\pi}\right) \Gamma\left(1-\frac{1}{2}\left(\frac{\theta}{\pi}-\frac{i \pi}{g}\right)\right) \Gamma\left(1-\frac{1}{2}\left(\frac{\theta}{\pi}+\frac{i \pi}{g}\right)\right)}{\Gamma\left(1-\frac{\theta}{\pi}\right) \Gamma\left(\frac{1}{2}\left(\frac{\theta}{\pi}+\frac{i \pi}{g}\right)\right) \Gamma\left(\frac{1}{2}\left(\frac{\theta}{\pi}-\frac{i \pi}{g}\right)\right)}\right. \\
& \left.\times \mathrm{e}^{-g\left(1-\frac{\theta}{\pi}\right) \alpha}{ }_{4} F_{3}\left(\begin{array}{c}
\beta_{1}, \beta_{2}, \beta_{2}, \beta_{1} \\
1-\frac{\theta}{\pi}, 1,1-\frac{\theta}{\pi}
\end{array} ; \exp (-2 g \alpha)\right)\right) \\
+ & \left(\frac{\Gamma\left(-\frac{\theta}{\pi}\right) \Gamma\left(1+\frac{1}{2}\left(\frac{\theta}{\pi}-\frac{i \pi}{g}\right)\right) \Gamma\left(1+\frac{1}{2}\left(\frac{\theta}{\pi}+\frac{i \pi}{g}\right)\right)}{\Gamma\left(1+\frac{\theta}{\pi}\right) \Gamma\left(\frac{1}{2}\left(\frac{i \pi}{g}-\frac{\theta}{\pi}\right)\right) \Gamma\left(\frac{1}{2}\left(-\frac{i \pi}{g}-\frac{\theta}{\pi}\right)\right)}\right. \\
& \left.\times \mathrm{e}^{-g\left(1+\frac{\theta}{\pi}\right) \alpha}{ }_{4} F_{3}\left(\begin{array}{c}
\gamma_{1}, \gamma_{2}, \gamma_{2}, \gamma_{1} \\
1+\frac{\theta}{\pi}, 1,1+\frac{\theta}{\pi}
\end{array} \exp (-2 g \alpha)\right)\right),
\end{aligned}
$$

where

$$
\begin{array}{ll}
\beta_{1}=1-\frac{1}{2}\left(\frac{\theta}{\pi}-\frac{i \pi}{g}\right), & \beta_{2}=1-\frac{1}{2}\left(\frac{i \pi}{g}+\frac{\theta}{\pi}\right) \\
\gamma_{1}=1+\frac{1}{2}\left(\frac{\theta}{\pi}-\frac{i \pi}{g}\right), & \gamma_{2}=1+\frac{1}{2}\left(\frac{\theta}{\pi}+\frac{i \pi}{g}\right) .
\end{array}
$$

As $\alpha \rightarrow \infty$,

$$
\operatorname{tr} \mathcal{L}_{[\alpha, \infty)} \sim C(g) \exp \left(-2 g\left(1-\frac{\theta}{\pi}\right) \alpha\right)
$$

where

$$
C(g):=\left(\frac{\Gamma\left(\frac{\theta}{\pi}\right) \Gamma\left(1-\frac{1}{2}\left(\frac{\theta}{\pi}-\frac{i \pi}{g}\right)\right) \Gamma\left(1-\frac{1}{2}\left(\frac{\theta}{\pi}+\frac{i \pi}{g}\right)\right)}{\left(1-\frac{\theta}{\pi}\right) \Gamma\left(1-\frac{\theta}{\pi}\right) \Gamma\left(\frac{1}{2}\left(\frac{\theta}{\pi}+\frac{i \pi}{g}\right)\right) \Gamma\left(\frac{1}{2}\left(\frac{\theta}{\pi}-\frac{i \pi}{g}\right)\right)}\right)^{2} .
$$


We have obtained (2.27) from (2.19) and the trace. Note that since $0<\theta / \pi<1 / 2$, the leading exponential factor, $\exp (-g(1+\theta / \pi) \alpha)$, in the second term of $(2.26)$ will tend to zero faster than the first. Furthermore, since

$$
{ }_{4} F_{3}\left(\begin{array}{l}
A_{1}, \ldots, A_{4} \\
B_{1}, \ldots, B_{3}
\end{array} ; z\right)=1+\sum_{n=0}^{\infty} \delta_{n} z^{n}, \quad|z|<1,
$$

where the above series converges absolutely in the unit circle, we see that only the exponential factor in the first term of (2.26) will be relevant in the computation of $\operatorname{tr} \mathcal{L}_{[\alpha, \infty)}$ for large $\alpha$.

Here we give an expression for $\operatorname{tr} \mathcal{L}_{[\alpha, \infty)}^{k}$, where $k$ is any positive integer and with this determine the higher order terms for large $\alpha$. We can compute $\operatorname{det}\left(I-\mathcal{L}_{[\alpha, \infty)}\right)$ by computing traces. The justification for this fact follows from our estimate for $f(x)$ when $x$ is large. It shows that the function is exponentially small. Hence when $x$ is larger than $\alpha$ it follows that the operator $\mathcal{L}_{[\alpha, \infty)}$ has norm smaller than one.

To compute the traces we note that from the expression of $\mathcal{L}(x, y)$ given by (2.19) and by shifting the interval of integration, $[\alpha, \infty)$, in the trace to $[0, \infty)$,

$$
\operatorname{tr} \mathcal{L}_{[\alpha, \infty)}^{k}=\int_{0}^{\infty} \ldots \int_{0}^{\infty} f\left(x_{1}+x_{2}+\alpha\right) \ldots f\left(x_{2 k}+x_{1}+\alpha\right) d x_{1} \ldots d x_{2 k} .
$$

For fixed $x, y>0$, as $\alpha \rightarrow \infty$,

$$
f(x+y+\alpha) \sim 2 g \frac{\Gamma\left(\frac{\theta}{\pi}\right) \Gamma\left(1+\frac{1}{2}\left(\frac{\theta}{\pi}-\frac{i \pi}{g}\right)\right) \Gamma\left(1-\frac{1}{2}\left(\frac{\theta}{\pi}+\frac{i \pi}{g}\right)\right)}{\Gamma\left(1-\frac{\theta}{\pi}\right) \Gamma\left(\frac{1}{2}\left(\frac{\theta}{\pi}+\frac{i \pi}{g}\right)\right) \Gamma\left(\frac{1}{2}\left(\frac{\theta}{\pi}-\frac{i \pi}{g}\right)\right)} \mathrm{e}^{-g\left(1-\frac{\theta}{\pi}\right)(x+y+\alpha)},
$$

where the second exponentially decaying term in (2.26) has been discarded. Integrating (2.29) with (2.30) we find, as $\alpha \rightarrow \infty$,

$$
\operatorname{tr} \mathcal{L}_{[\alpha, \infty)}^{k} \sim C(g)^{k} \exp \left(-2 g\left(1-\frac{\theta}{\pi}\right) k \alpha\right)
$$

Therefore,

$$
\begin{aligned}
-\ln \operatorname{det}\left(I-\mathcal{L}_{[\alpha, \infty)}\right) & =\sum_{k=1}^{\infty} \frac{\operatorname{tr} \mathcal{L}_{[\alpha, \infty)}^{k}}{k} \\
& \sim \sum_{k=1}^{\infty} \frac{C^{k}(g)}{k} \mathrm{e}^{-2 g\left(1-\frac{\theta}{\pi}\right) \alpha k} .
\end{aligned}
$$

Finally,

$$
\operatorname{det}\left(I-\mathcal{L}_{[\alpha, \infty)}\right) \sim 1-C(g) \mathrm{e}^{-2 g \alpha(1-\theta / \pi)}, \quad \alpha \rightarrow \infty
$$

In random matrix theory $E(\alpha):=\operatorname{det}\left(I-\mathcal{K}_{[0, \alpha]}\right)$ is the probability that an interval $[-\alpha / 2, \alpha / 2]$ (after a suitable scaling) is free of eigenvalues. Putting $g=0$, in (2.1), we have the sine 
kernel, $\frac{\sin (\pi(x-y))}{\pi(x-y)}$. It was shown in [5] that $R(\alpha):=-\frac{d \ln E(\alpha)}{d \alpha}$, satisfies a particular Painléve $\mathrm{V}$ equation. For a simpler derivation of this and a review of random matrix theory see [10]. Recently, it was shown in [9] using the theory of [11] that $R(\alpha)$ for $g>0$, satisfies a particular Painléve VI. In a heuristic perturbative calculation on the Painléve VI both the exponential decaying terms were was found [7], however, the prefactor, $C(g)$, cannot be determined as it involves an indefinite integral. It appears that $C(g)$ can only be obtained from determinant identity, (1.3). It can also be shown that the computation of (1.1) with the kernel given by (2.13) can be reformulated as an equivalent $2 \times 2$ matrix Riemann-Hilbert problem, see [6] for a description of this technique. Although as $\alpha \rightarrow \infty$, such a technique could reproduce the classical results of Akhiezer, Hirschman and Szegö, namely, the $Z$ factor, it is not at all clear whether the higher correction terms given by (2.34) can be found. Final remark: the determinant identity, (1.3), does not hold for $g=0$. Since in this situation, the Fourier transform of the sine kernel is the characteristic function of $[-\pi / 2, \pi / 2]$ and the Wiener-Hopf factorization fails.

\section{References}

[1] E. Basor, H Widom, On a Toeplitz Identity of Borodin and Okounkov, Integral Equations and Operator Theory, 37, (2002) 397-401.

[2] I.S. Gradshteyn, I.M. Ryzhik, Table of integrals, series, and products, Fifth Edition, Academic Press, New York, 1994.

[3] A. Böttcher, On the determinant formulas by Borodin, Okounkov, Baik, Deift and Rains, FA/0101008.

[4] A. Böttcher and B. Silbermann, Analysis of Toeplitz Operators, Springer-Verlag, Berlin, 1990.

[5] M. Jimbo, T. Miwa, Y. Mori and M. Sato, Density matrix of an inpenetrable Base gas and the fifth Painlevé transcendent, Physica 1D, (1980) 80-158.

[6] V. E. Korepin, N. M. Bogoliubov and A. G. Izergin, Quantum inverse scattering methods and correlation functions, Cambridge University Press, 1997.

[7] N. D. Lawrence, Random Matrices and Orthogonal Polynomials, Ph. D. Thesis, University of London, 2001, Chapter 4, unpublished.

[8] K. A. Muttalib, Y. Chen, M. E. H. Ismail and V. N. Nicopoulos, A New Family of Unitary Random Matrices, Phys. Rev. Lett., 71, (1993) 471-475. 
[9] S. M. Nishigaki, Level spacing distribution of critical random matrix ensembles, Phys. Rev. E 58 (1998) 6915-6918.

[10] C. A. Tracy and H. Widom, Introduction to random matrices in Geometric and quantum aspects of integrable systems, ed. G. F. Helminck, Springer Lecture Notes in Physics Vol. 424 (1993) 103-130.

[11] C. A. Tracy and H. Widom, Fredholm determinants, differential equations and matrix models, Commun. Math. Phys. 163 (1994) 289-310.

Department of Mathematics Department of Mathematics

California Polytechnic State University Imperial College

San Luis Obispo, CA 93407 USA 180 Queen's gate, London, SW7 2BZ, UK

ebasor@calpoly.edu $\quad$ y.chen@ic.ac.uk 